\title{
Direct observation of the coherent precession of magnetic domain walls propagating along permalloy nanowires
}

\author{
MASAMITSU HAYASHI ${ }^{1,2}$, LUC THOMAS ${ }^{1}$, CHARLES RETTNER ${ }^{1}$, RAI MORIYA ${ }^{1}$ AND STUART S. P. PARKIN ${ }^{1 *}$ \\ ${ }^{1}$ IBM Research Division, Almaden Research Center, San Jose, California 95120, USA \\ ${ }^{2}$ Department of Materials Science and Engineering, Stanford University, Stanford, California 94305, USA \\ *e-mail: parkin@almaden.ibm.com
}

The dynamics of the motion of domain walls (DWs) in magnetic materials has been extensively explored theoretically ${ }^{1-3}$. Depending on the driving force, conventionally magnetic field and, more recently, spin-polarized current ${ }^{4-13}$, the propagation of DWs changes from a simple translation to more complex precessional modes ${ }^{14}$. Experimentally, indirect evidence of this transition is found from a sudden drop in the wall's velocity ${ }^{15-18}$, but direct observation of the precessional modes is lacking. Here we show experimentally, using a combination of quasi-static and real-time measurement techniques, that DWs propagate along permalloy nanowires with a periodic variation in the chirality of the walls. The frequency of this oscillation is consistent with a precession of the propagating DW, increasing linearly with field according to the Larmor precession frequency. Current in the nanowire, large enough to significantly influence the DW velocity $^{18,19}$, has little effect on the precession frequency but can be used to adjust the phase of the wall's precession. The highly coherent and reproducible motion of the DW revealed by our studies demonstrates that the DW is a well-defined macroscopic object whose phase is inextricably interlinked to the distance travelled by the DW.

With the advent of magnetic nanowires of dimensions comparable to magnetic DW widths, it is possible to imagine that DWs propagating along such wires will exhibit a well-defined precessional mode due to confinement. In contrast, in extended structures, which have been extensively studied in the past, many modes are accessible ${ }^{1,20}$. Permalloy nanowires are attractive because they exhibit large anisotropic magnetoresistance (AMR) so that transverse and vortex walls can be readily detected and identified by resistance measurements. Furthermore, by breaking the onedimensional symmetry of the nanowire with a notch along one of its edges, the chirality of the DW can also be discerned ${ }^{21}$. Here, notched nanowires are used to directly show that the chirality of transverse DWs periodically reverses as the walls propagate in the precessional regime. This precessional motion is also detected in real time using time-resolved resistance measurements, which show that the precessional motion is, surprisingly, highly coherent.

A scanning electron microscopy image of a typical permalloy nanowire with two contact lines, labelled $\mathrm{A}$ and $\mathrm{B}$, is shown in Fig. 1a. Using a suitable field sequence (see the Methods section), a DW is introduced into section A-B of the nanowire by injecting a voltage pulse into contact line A. A magnetic field, $H$, is applied along the wire during the voltage pulse injection to assist the subsequent propagation of a DW. A fraction of the injected voltage pulse flows into section $A-B$, thereby injecting current into the nanowire as the DW propagates along it. The current density that flows into the nanowire scales as $\sim 0.5 \times 10^{8} \mathrm{~A} \mathrm{~cm}^{-2} \mathrm{~V}^{-1}$. Positive current is defined as current flowing from line $\mathrm{A}$ to $\mathrm{B}$.

The existence of a DW in section A-B can readily be monitored by measuring the resistance of the device. Figure $1 b$ shows a histogram of the resistance of the nanowire after a DW is injected, using a $2.5 \mathrm{~V}, 10$-ns-long voltage pulse, and trapped at a pinning site (that is, a notch) located within section A-B. Here $\Delta R$ represents the difference in the nanowire resistance before and after the DW injection. Owing to the AMR effect, the resistance level decreases when a DW is present, resulting in a negative $\Delta R$. Zero $\Delta R$ represents the state without any DW in section $\mathrm{A}-\mathrm{B}$. Three distinct non-zero $\Delta R$ values are observed in the histogram, whose corresponding magnetic structures, imaged using magnetic force microscopy (MFM), are shown in Fig. 1b. Two transverse walls with clockwise, $T_{\mathrm{C}}$, and anticlockwise, $T_{\mathrm{A}}$, chiralities and one clockwise vortex wall, $V_{\mathrm{C}}$, are observed. A small peak around $\Delta R \sim-0.29$ presumably corresponds to the anticlockwise vortex wall. The probability of injecting and trapping each type of DW at the notch is plotted as a function of $H$ in Fig. 1c. The probability of injecting different types of DWs has a complex dependence on the magnetic field. Vortex walls can be injected and trapped in a relatively small field range with much lower probability than either of the two transverse walls. Note that the dimensions of the nanowire used here favour transverse walls.

The probabilities of trapping $T_{\mathrm{A}}, T_{\mathrm{C}}$ and $V_{\mathrm{C}}$ DWs at the notch are plotted as a function of the pulse length for negative and positive voltage pulses in Fig. $2 \mathrm{a}$ and $\mathrm{b}$, respectively, for several fields. For the case of positive voltages, oscillations in the trapping probability are observed for pulse lengths up to $\sim 100 \mathrm{~ns}$, the limit of our experimental setup. In contrast, for negative voltages, oscillations in the probability are seen only for much shorter pulse lengths, typically below $\sim 30 \mathrm{~ns}$. In both cases, oscillations are clearly observed only for fields above $\sim 14$ Oe, which is close to the Walker breakdown field for these nanowires. Below this field, the trapping probability is nearly independent of the pulse length. The Walker breakdown ${ }^{22}$ can be observed in the DW velocity versus field plot (see description of the measurements below), as shown in Fig. 2c. Note that for large positive voltages at lower fields, the pressure from current, via spin-transfer torque, compensates the pressure from the field, which can result in $\sim$ zero DW velocity ${ }^{18}$. 

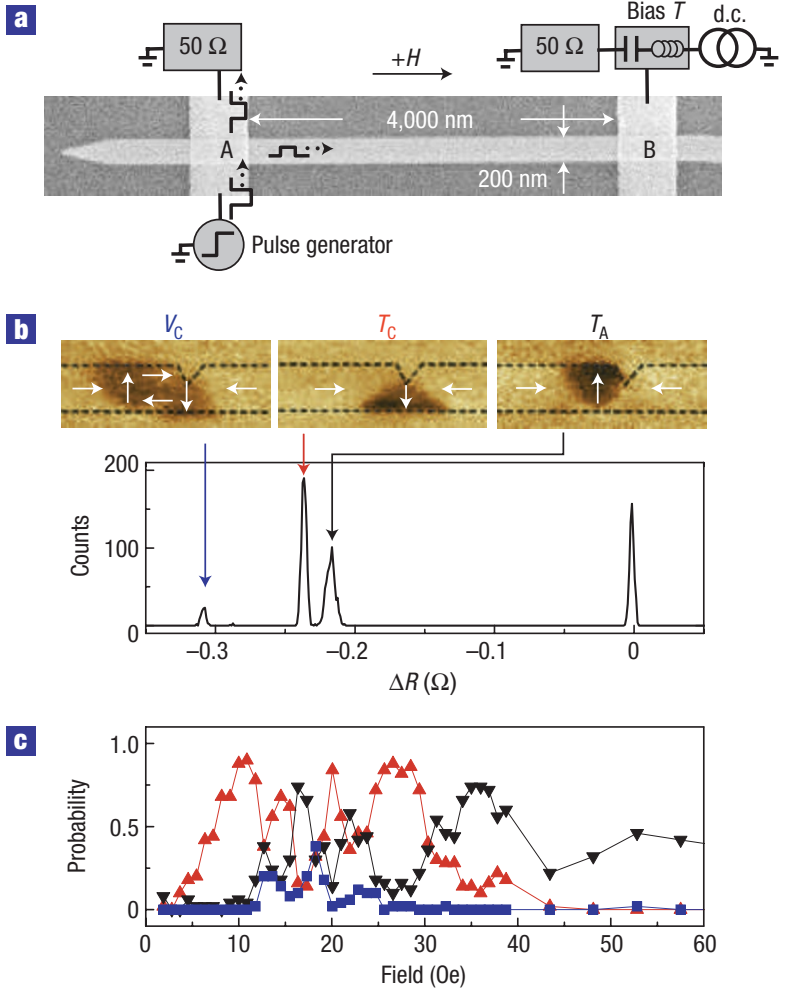

Figure 1 Experimental set-up and structure of injected DWs at a notched pinning site. a, Scanning electron microscopy image of a permalloy nanowire (horizontal) and its electrical contacts (vertical lines). Note that there is no notched pinning site in the nanowire shown in this image. A schematic illustration of the quasi-static resistance measurement set-up is overlaid on the image. $\mathbf{b}$, Histogram of $\Delta R$ values measured in successive repeated experiments in which a DW is injected into the nanowire using a 2.5-V-high, 10 -ns-long voltage pulse and is trapped at a notch located $\sim 3 \mu \mathrm{m}$ away from line $A$. The notch is triangular, as shown in the MFM images above. $\Delta R$ is the difference in the resistance of the nanowire before and after the DW injection. The MFM images show the magnetic configurations corresponding to each $\Delta R$ peak. The white arrows denote the magnetization directions. c, Probability of trapping a DW at the notch for $V_{\mathrm{C}}$ (blue), $T_{\mathrm{C}}$ (red) and $T_{\mathrm{A}}$ (black) walls as a function of the magnetic field. A 2.5V, 10-ns-long voltage pulse is used to inject a DW.

The oscillation frequency, $f_{\mathrm{QS}}$, that is, the inverse of the oscillation period, and the phase of the trapping probabilities are plotted in Fig. 2d and e, respectively, for each DW state. Whereas $f_{\mathrm{QS}}$ is approximately proportional to $H$ regardless of the DW state, the phases of the oscillations depend on the DW state. $T_{\mathrm{A}}$ and $T_{\mathrm{C}}$ walls are $180^{\circ}$ out of phase with each other and the $V_{\mathrm{C}}$ wall is $90^{\circ}$ out of phase from the transverse walls. At higher fields, above $\sim 40$ Oe, only $T_{\mathrm{A}}$ walls are trapped at the notch: in this field range we find that $f_{\mathrm{OS}}$ saturates and the phase of the oscillation varies little.

The pulse length dependence of the trapping probabilities for the transverse DWs are shown in Fig. 3a for different pulse amplitudes at a fixed field. The extracted oscillation frequencies and phases for the $T_{\mathrm{A}}$ DW are plotted in Fig. $3 \mathrm{~b}$ and $\mathrm{c}$ as a function of the pulse amplitude, respectively. At a fixed field, the oscillation frequency is nearly independent of the amplitude, whereas the phase depends on the pulse amplitude.

The oscillatory variation observed in the trapped DW structure with pulse length strongly suggests that the DW structure evolves during its propagation. We explored this possibility by carrying out real-time resistance measurements of the nanowire during the DW propagation ${ }^{18}$. These measurements were carried out on a permalloy wire without any artificial pinning sites. The DW injection and propagation experiment is repeated more than 16,000 times and the signal traces from each of these experiments are averaged to obtain an adequate signal-to-noise ratio. Figure 4a,b shows typical traces of the signal $\Delta V$ obtained by the oscilloscope. This signal, which is proportional to $\Delta R$, is increased for the period of time $\Delta \tau$ for which the DW spends in section A-B. $\Delta \tau$ depends on the DW velocity, which is influenced by the current due to spintransfer torque. This is clearly seen by the DW velocity, equal to $L(\sim 4 \mu \mathrm{m}) / \Delta \tau$, plotted in Fig. $2 \mathrm{c}$. Negative and positive currents increase and decrease the DW velocity, respectively ${ }^{18,19}$.

Oscillations of the signal amplitude can be seen, for both polarities of the voltage pulse, when the DW is propagating along section A-B. This is particularly remarkable given that the signal represents an average of more than 16,000 successive independent DW injections and propagations along the nanowire. Thus, this entire process must be highly repeatable and coherent.

Exemplary fast Fourier transform (FFT) spectra of these traces are shown in Fig. 4c, inset. In these spectra, a well-defined peak is observed at a frequency, $f_{\mathrm{D}}$. Plots of $f_{\mathrm{D}}$ versus $H$, which are shown in Fig. 4c, show that $f_{\mathrm{D}}$ is linearly proportional to $H$, for both voltage polarities. Whereas $f_{\mathrm{D}}$ varies strongly with $H$, it has a weak dependence on current, as shown by the plots of $f_{\mathrm{D}}$ versus voltage amplitude in Fig. $4 \mathrm{~d}$. We do find, however, that $f_{\mathrm{D}}$ is systematically higher for negative as compared with positive currents of the same magnitude. Note that the frequency obtained in this dynamic measurement is approximately twice that found in the quasi-static measurements at the same field (see Fig. 2d). Also, consistent with the quasi-static studies, oscillations are observed only when the field exceeds the Walker breakdown field $(\sim 14 \mathrm{Oe})$.

We first use micromagnetic simulations ${ }^{23}$ (see the Methods section) to identify the origin of the oscillations of the DW resistance seen in the dynamic measurements. Figure 5 a shows the temporal evolution of the DW position when a magnetic field of 20 Oe is applied (no current). The position of the DW oscillates as it propagates along the wire. These oscillations are associated with periodic changes in the DW structure, as indicated by the symbols $^{14}$. The DW state oscillates periodically from a transverse wall of one chirality to a transverse wall of the opposite polarity via a vortex wall or an anti-vortex wall state. Values of $\Delta R$ corresponding to these simulated magnetic configurations are shown in Fig. 5b. These can be compared with the experimentally measured signal traces shown in Fig. 4a,b. We thus conclude that the oscillations seen in the real-time measurements of the DW motion represent periodic variations in the $\mathrm{DW}$ structure as the $\mathrm{DW}$ propagates along the nanowire.

The quasi-static results can be understood within the same framework. The structure of the DW will evolve periodically during the time that the DW travels from its point of injection to the pinning centre $(\sim 3 \mu \mathrm{m})$. This time is determined by the average DW velocity, which depends on the field and the duration and magnitude of the current pulse. Thus, for a given field and current pulse amplitude, the DW will arrive at the notch sooner when the spin-polarized current drives the DW towards the notch and later when the current is in the opposite direction. For a given current direction, the arrival time of the DW is then determined by the length of the current pulse unless the pulse is so long that the DW reaches the notch before the end of the pulse. On the other hand, the frequency of the periodic structural changes is largely independent of the current (see Fig. 4d). Therefore, the DW will have a distinct structure when it arrives at the pinning centre, oscillating with the pulse length. For positive voltages, when the current opposes the field-driven motion of the DW, the 

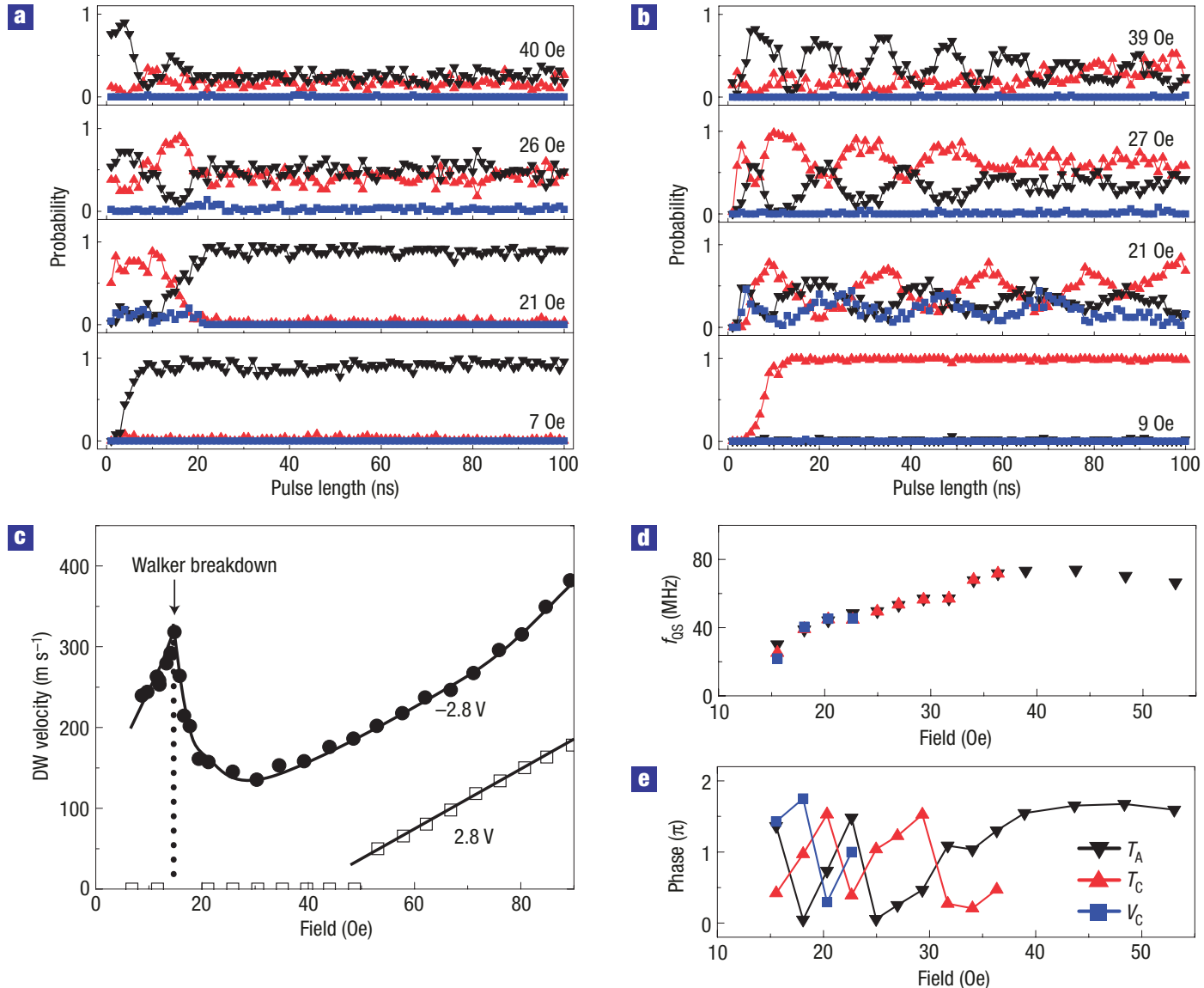

Figure 2 Probability of trapping DWs with different structures at a pinning site: field dependence. a,b, Probability of trapping a DW, $V_{\mathrm{C}}$ (blue), $T_{\mathrm{C}}$ (red) and $T_{\mathrm{A}}$ (black), at a notch plotted against the voltage pulse length at several different fields, when $-2.5 \mathrm{~V}$ (a) and $2.5 \mathrm{~V}$ (b) pulses are used to inject a DW, respectively. c, DW velocity versus magnetic field measured using $-2.8 \mathrm{~V}$ (filled circles) and $2.8 \mathrm{~V}$ (open squares) pulses to inject the DW. The Walker breakdown field is indicated. Note that our experimental set-up is limited to measurements of DW velocities above $40 \mathrm{~m} \mathrm{~s}^{-1}$. Velocities below this limit are shown as zero velocities. d,e, Frequency $f_{0 \mathrm{~S}}$ (d) and phase (e) obtained from fitting the oscillatory dependence of trapping probability on pulse length curves (typical curves shown in $\mathbf{b}$ ) to an $\operatorname{exponential~sinusoid~of~the~form,~} A$ exp $\left(-t_{\mathrm{p}} / \tau_{\mathrm{D}}\right.$ ) $\sin \left(2 \pi f_{0 \mathrm{~S}} t_{\mathrm{p}}+\right.$ phase), where $t_{\mathrm{p}}$ is the pulse length, $A$ is the amplitude and $\tau_{\mathrm{D}}$ is the decay time. $f_{\mathrm{QS}}$ and the phase are plotted against the magnetic field for each DW structure. The data shown correspond to the case when a $2.5 \mathrm{~V}$ pulse is used to inject the DW.
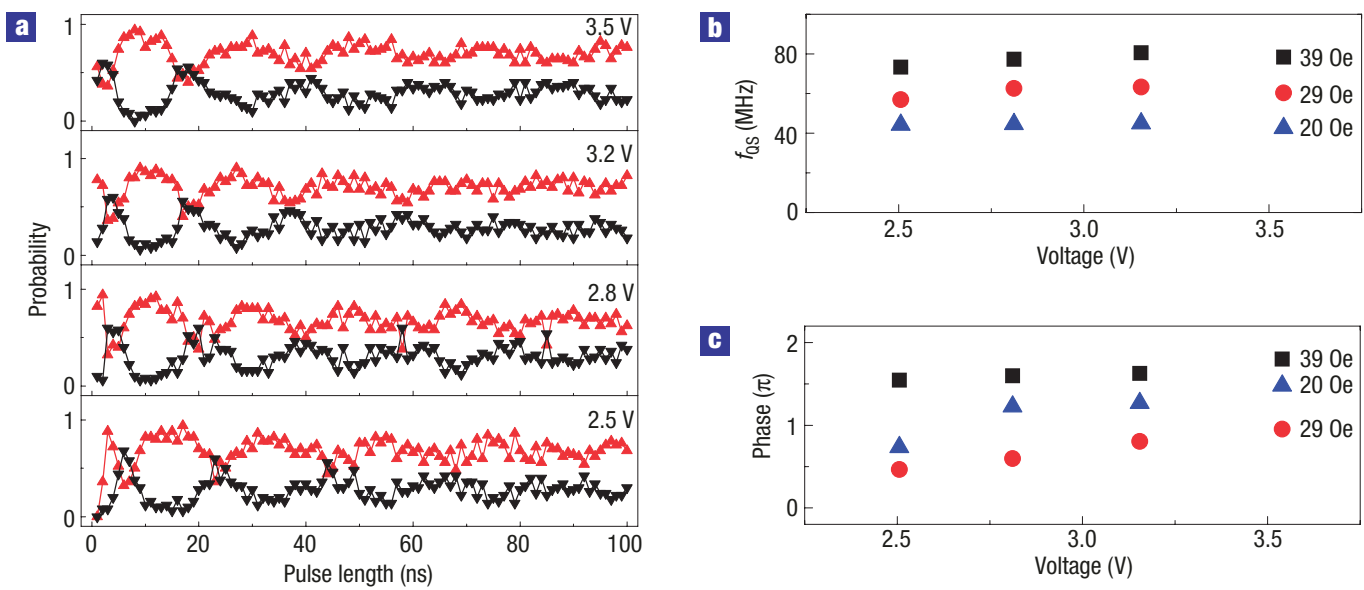

Figure 3 Probability of trapping transverse DWs with different chiralities at a pinning site: voltage pulse dependence. a, Probability of trapping a transverse wall, (red) and $T_{\mathrm{A}}$ (black), at a notch plotted against the voltage pulse length for various pulse amplitudes. The applied magnetic field is $250 \mathrm{e}$. b,c, Dependence of the frequency $f_{\mathrm{QS}}(\mathbf{b})$ and the phase shift (c) of the oscillations of the trapping probability on the pulse amplitude. The method of deducing of $f_{\mathrm{aS}}$ and the phase shift are described in the Fig. $2 \mathrm{~d}$ caption. The corresponding magnetic fields used during the injection of a DW are shown in the panels. 

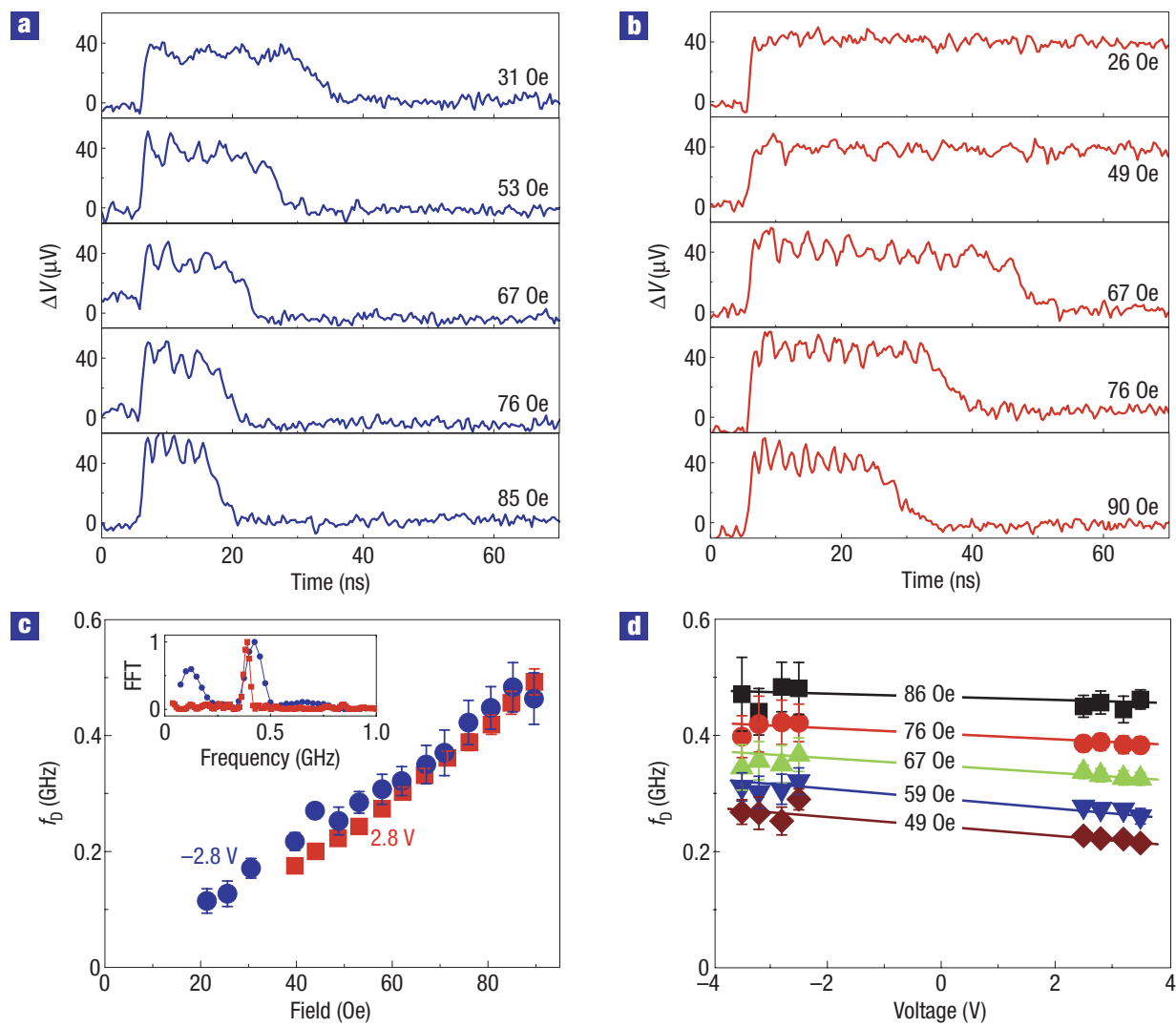

Figure 4 Time-resolved resistance measurements of a propagating DW along a permalloy nanowire. a,b, Real-time measurements of the DW propagation along the nanowire obtained by averaging the temporal evolution of the nanowire resistance 16,000 times. Signal traces $\Delta V$ obtained by using $-2.8 \mathrm{~V}$ (a) and $2.8 \mathrm{~V}$ (b) voltage pulses to inject a DW. Representative signal traces are shown at various fields indicated in each panel. c, Dependence of the frequency of the oscillations in resistance observed in the signal traces $(\Delta V)$ plotted versus field. The data shown are when $\pm 2.8 \mathrm{~V}$ voltage pulses are used to inject a DW. Oscillation frequency $f_{\mathrm{D}}$ is determined by taking the FFT spectra of each trace. Error bars correspond to the width of a gaussian to which the peak structure in the FFT spectra is fitted. The inset shows normalized FFT spectra of the signal traces taken at $760 \mathrm{e}$. Note that the lower-frequency feature simply corresponds to $1 / \Delta \tau$. $\mathbf{d}$, Dependence of the oscillation frequency $f_{\mathrm{D}}$ on the amplitude of the voltage pulse in various magnetic fields. The definition of the error bars is the same as in c. The solid lines are guides to the eye.

DW moves so slowly that it never reaches the pinning site before the end of the 100-ns-long voltage pulse used. Thus, we observe oscillations in DW structure for pulses up to $100 \mathrm{~ns}$ long as shown in Fig. 2b. This interpretation assumes that the DW structure does not evolve further once the DW is pinned at the notch. This assumption is consistent with the quasi-static results for negative currents, in which the DW moves faster as the current pushes it towards the notch. In this case, when the current pulse exceeds the time for the DW to reach the notch $(\sim 15-30 \mathrm{~ns}$ in Fig. 2a), the trapping probability becomes constant, which would not be the case if the DW structure continued to evolve once pinned. Note, however, that slightly higher current densities can result in DW transformations ${ }^{21}$.

The periodic variation in DW structure thus accounts for both the oscillatory dependence of the DW trapping probability on current pulse length and the oscillations in DW resistance during motion. Moreover, the difference by a factor of two in frequencies of these oscillations can be readily understood because in the quasi-static measurements the $T_{\mathrm{A}}$ and $T_{\mathrm{C}}$ walls have different resistance values, whereas in the dynamic measurements they have the same resistance. This is because the notch used in the quasi-static experiments is asymmetric so that the $T_{\mathrm{A}}$ and $T_{\mathrm{C}}$ walls are distinguishable. These oscillation frequencies are compared in Fig. $5 c$, where $f_{\mathrm{D}}$ and $2 f_{\mathrm{QS}}$ are plotted. At low fields the frequencies are in reasonable agreement but presumably at higher fields the notch potential plays a significant role so accounting for the difference.

Finally, it is interesting to compare $f_{\mathrm{D}}$ with the oscillation frequency calculated using the well-known one-dimensional model of DW motion ${ }^{1,2,22}$. This frequency, in the absence of current, is given by $f_{\mathrm{lD}}=(\gamma / \pi) \sqrt{H^{2}-H_{\mathrm{WB}}^{2}} /\left(1+\alpha^{2}\right)$, where $\gamma$ is the gyromagnetic ratio, $\alpha$ is the Gilbert damping constant and $H_{\mathrm{WB}}$ is the Walker breakdown field (see the Methods section). The slope of the frequency versus field curve is given simply by $\sim \gamma / \pi$, which is two times the Larmor frequency, in excellent agreement with our real-time measurements, as shown by the solid line in Fig. 5c.

\section{METHODS}

\section{SAMPLE DESCRIPTION}

Permalloy nanowires are formed from films of $0.5 \mathrm{Fe} / 10 \mathrm{AlOx} / 10 \mathrm{Ni}_{81} \mathrm{Fe}_{19} / 0.5$ $\mathrm{TaN} / 5 \mathrm{Ru}$ (units in nanometres), deposited on high-resistive Si substrates by magnetron sputter deposition. Electron beam lithography and Ar ion etching are used to pattern $\sim 200$-nm-wide nanowires and to form electrical contacts formed from films of $5 \mathrm{~nm} \mathrm{Ta} / 45 \mathrm{~nm} \mathrm{Rh}$. High-bandwidth $(40 \mathrm{GHz})$ probes are used to make electrical contacts to the devices.

A DW is injected into the nanowire by using the following method ${ }^{18,21}$. First, the magnetization of the nanowire is set along one direction by applying a 

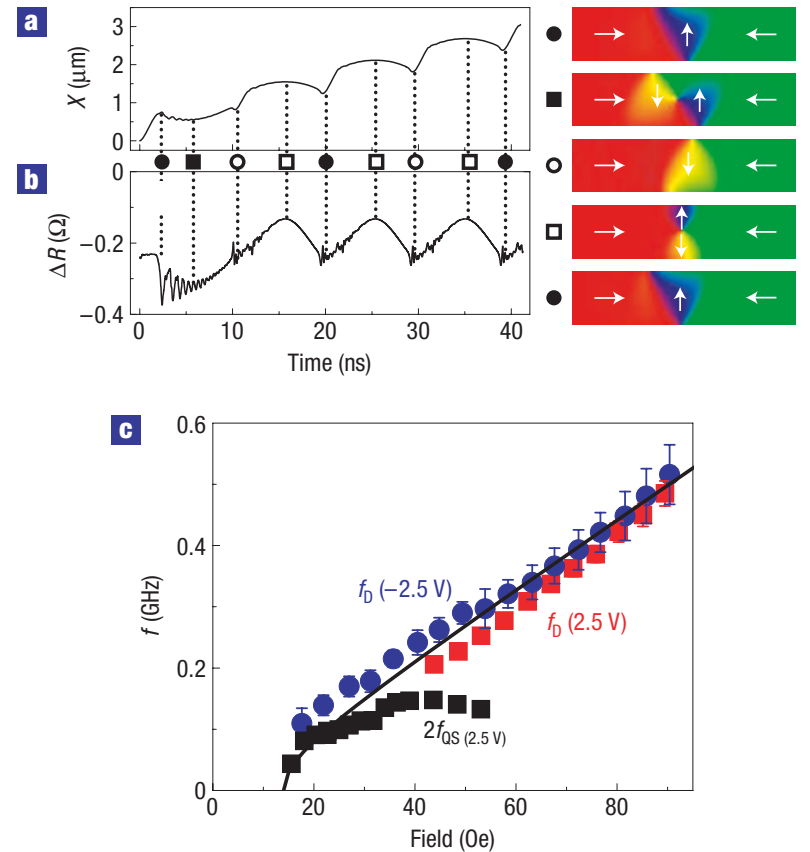

Figure 5 Micromagnetic simulations of the field-driven motion of a DW and comparison of calculated and measured frequency of periodic DW motion. $\mathbf{a}, \mathbf{b}$, Temporal evolution of the position of the DW (a) and the estimated $\Delta R(\mathbf{b})$ of the nanowire calculated from micromagnetic simulations in an applied field of $200 \mathrm{e}$ and zero current. The sequence of simulated DW configurations at the extrema of the first cycle of its periodic motion along the nanowire are shown at the right hand side of the figure (from top to bottom with increasing time). The symbols correspond to time-stamps. The white arrows denote the magnetization directions in the simulated images. c, Dependence of the oscillation frequency, $f_{\mathrm{D}}$, on magnetic field when $\pm 2.5 \mathrm{~V}$ voltage pulses are used to inject a DW. The definition of the error bars are the same as in Fig. $4 \mathrm{c}$ caption. These data are compared with the oscillation frequency $f_{\mathrm{QS}}$ obtained from the quasi-static experiments shown in Fig. 2d. The solid line represents values of $f_{D}$ estimated from the one-dimensional model in zero current, where a value of $\alpha=0.01$ is used.

global-reset magnetic field of $\sim 300$ Oe along the nanowire. Then the direction of this field is reversed to provide a global-assist field $H$ during the subsequent injection and propagation of a DW along the nanowire. With $H$ turned on, a voltage pulse, varying in length from 1-100 ns, is injected into contact line A. This pulse generates a highly localized magnetic field under line A, which creates a DW at each edge of this line. The DW created in section A-B will move towards line B under the influence of $H$.

For the quasi-static measurements, a permalloy nanowire with an artificial pinning site patterned $\sim 3 \mu \mathrm{m}$ away from line $\mathrm{A}$ is used. The pinning site consists of a triangle-shaped notch on one side of the nanowire, whose depth is $\sim 30 \%$ of the wire width. For the time-resolved resistance measurements, permalloy nanowires without any artificial pinning sites are used.

\section{MICROMAGNETIC SIMULATION}

Micromagnetic simulations were carried out on permalloy nanowires with the same physical dimensions as those used in the experiments $(200 \mathrm{~nm}$ wide, $10 \mathrm{~nm}$ thick), for fields above the Walker breakdown field. Moving boundary conditions are used to avoid end effects. The Gilbert damping parameter $\alpha$ is set to 0.01 to match the experimental value of the Walker breakdown field. A transverse wall with anticlockwise chirality (top panel of the images shown at the right-hand side of Fig. $5 \mathrm{a}, \mathrm{b}$ ) is chosen as the initial state to compute the temporal evolution of the position and the structure of the DW driven by magnetic field, where the results are shown in Fig. 5a. To estimate $\Delta R$, the resistivity and the AMR ratio of the permalloy nanowire are set to $\sim 30 \mu \Omega \mathrm{cm}$ and $1.5 \%$, respectively.

\section{ONE-DIMENSIONAL MODEL}

The differential equations of the one-dimensional model of a DW, including the spin-transfer torque terms ${ }^{10,11,13}$, are solved analytically to obtain the period of the oscillation of the DW position. The oscillation frequency is given by

$$
f_{1 \mathrm{D}}=\frac{\gamma}{\pi} \frac{\sqrt{[H+(\beta-\alpha) u / \gamma \Delta]^{2}-H_{\mathrm{WB}}^{2}}}{1+\alpha^{2}}
$$

where $u=\mu_{\mathrm{B}} J P / e M_{\mathrm{s}}, \mu_{\mathrm{B}}$ is the Bohr magnetron, $J$ is the current density, $P$ is the spin polarization of the current, $e$ is the electric charge, $M_{\mathrm{s}}$ is the saturation magnetization, $\Delta$ is the DW width and $\beta$ is the non-adiabatic spin-torque term. The sign of $u$ is set to be positive when the electron flow acts in concert with the field on the DW. Setting the current to zero $(u=0)$ gives the expression shown in the text.

\section{Received 29 August 2006; accepted 23 October 2006; published 3 December 2006.}

References

1. Malozemoff, A. P. \& Slonczewski, J. C. Magnetic Domain Walls in Bubble Material (Academic, New York, 1979).

2. Bar' yakhtar, V. G., Chetkin, M. V., Ivanov, B. A. \& Gadetskii, S. N. Dynamics of Topological Magnetic Solitons (Springer, Berlin, 1994).

3. Berger, L. Analysis of measured transport properties of domain walls in magnetic nanowires and films. Phys. Rev. B 73, 014407 (2006).

4. Berger, L. Exchange interaction between ferromagnetic domain-wall and electric-current in very thin metallic-films. J. Appl. Phys. 55, 1954-1956 (1984).

5. Yamaguchi, A. et al. Real-space observation of current-driven domain wall motion in submicron magnetic wires. Phys. Rev. Lett. 92, 077205 (2004).

6. Klaui, M. et al. Controlled and reproducible domain wall displacement by current pulses injected into ferromagnetic ring structures. Phys. Rev. Lett. 94, 106601 (2005).

7. Vernier, N., Allwood, D. A., Atkinson, D., Cooke, M. D. \& Cowburn, R. P. Domain wall propagation in magnetic nanowires by spin-polarized current injection. Europhys. Lett. 65, 526-532 (2004).

8. Klaui, M. et al. Direct observation of domain-wall configurations transformed by spin currents. Phys. Rev. Lett. 95, 026601 (2005).

9. Tatara, G. \& Kohno, H. Theory of current-driven domain wall motion: Spin transfer versus momentum transfer. Phys. Rev. Lett. 92, 086601 (2004).

10. Zhang, S. \& Li, Z. Roles of nonequilibrium conduction electrons on the magnetization dynamics of ferromagnets. Phys. Rev. Lett. 93, 127204 (2004).

11. Thiaville, A., Nakatani, Y., Miltat J. \& Suzuki, Y. Micromagnetic understanding of current-driven domain wall motion in patterned nanowires. Europhys. Lett. 69, 990-996 (2005).

12. Barnes, S. E. \& Maekawa, S. Current-spin coupling for ferromagnetic domain walls in fine wires. Phys. Rev. Lett. 95, 107204 (2005).

13. Thomas, L. et al. Oscillatory dependence of current-driven magnetic domain wall motion on current pulse length. Nature 443, 197-200 (2006).

14. Nakatani, Y., Thiaville, A. \& Miltat, J. Faster magnetic walls in rough wires. Nature Mater. 2, $521-523(2003)$

15. Zimmermann, L. \& Miltat, J. Instability of bubble radial motion associated with chirality changes. J. Magn. Magn. Mater. 94, 207-214 (1991).

16. Honda, S., Fukuda, N. \& Kusuda, T. Mechanisms of bubble-wall radial motion deduced from chirality switching and collapse experiments using fast-rise bias field pulse. J. Appl. Phys. 52, 5756-5762 (1981)

17. Beach, G. S. D., Nistor, C., Knutson, C., Tsoi, M. \& Erskine, J. L. Dynamics of field-driven domain-wall propagation in ferromagnetic nanowires. Nature Mater. 4, 741-744 (2005).

18. Hayashi, M. et al. Influence of current on field-driven domain wall motion in permalloy nanowires from time resolved measurements of anisotropic magnetoresistance. Phys. Rev. Lett. 96, 197207 (2006).

19. Beach, G. S. D., Knutson, C., Nistor, C., Tsoi, M. \& Erskine, J. L. Nonlinear domain-wall velocity enhancement by spin-polarized electric current. Phys. Rev. Lett. 97, 057203 (2006).

20. Slonczewski, J. C. Theory of domain-wall motion in magnetic-films and platelets. J. Appl. Phys. 44, 1759-1770 (1973).

21. Hayashi, M. et al. Dependence of current and field driven depinning of domain walls on their structure and chirality in permalloy nanowires. Phys. Rev. Lett. 97, 207205 (2006).

22. Schryer, N. L. \& Walker, L. R. Motion of 180 degrees domain-walls in uniform dc magnetic-fields. J. Appl. Phys. 45, 5406-5421 (1974).

23. Scheinfein, M. R. LLG micromagnetics simulator ${ }^{\mathrm{TM}}$. <http://llgmicro.home.mindspring.com/>

\section{Acknowledgements}

We thank DMEA for partial support of this work.

Correspondence and requests for materials should be addressed to S.S.P.P.

\section{Competing financial interests}

The authors declare that they have no competing financial interests.

Reprints and permission information is available online at http://npg.nature.com/reprintsandpermissions/ 